\title{
ON PRODUCTS OF PSEUDO-ANOSOV MAPS AND DEHN TWISTS OF RIEMANN SURFACES WITH PUNCTURES
}

\author{
C. ZHANG \\ (Received 21 October 2008; accepted 19 January 2010) \\ Communicated by P. C. Fenton
}

Abstract

Let $S$ be a Riemann surface of type $(p, n)$ with $3 p+n>4$ and $n \geq 1$. We investigate products of some pseudo-Anosov maps $\theta$ and Dehn twists $t_{\alpha}$ on $S$, and prove that under certain conditions the products $t_{\alpha}^{k} \circ \theta$ are pseudo-Anosov for all integers $k$. We also give examples that show that $t_{\alpha}^{k} \circ \theta$ are not pseudoAnosov for some integers $k$.

2000 Mathematics subject classification: primary 32G15; secondary 30F60.

Keywords and phrases: Riemann surfaces, Dehn twists, pseudo-Anosov maps, Teichmüller spaces.

\section{Introduction}

Let $S$ be a Riemann surface of type $(p, n)$, where $p$ is the genus and $n$ is the number of punctures of $S$. Assume that $3 p+n>3$. A nonperiodic map of $S$ onto itself is called reducible if it is isotopic to a map that keeps a system $\left\{c_{1}, \ldots, c_{s}\right\}$ of disjoint and independent simple curves on $S$ invariant. A map $f$ is called pseudoAnosov [5, 14] if it leaves invariant a pair of transverse measured foliations $\left\{\mathcal{F}_{+}, \mathcal{F}_{-}\right\}$ such that $f\left(\mathcal{F}_{+}\right)=\lambda \mathcal{F}_{+}$and $f\left(\mathcal{F}_{-}\right)=(1 / \lambda) \mathcal{F}_{-}$for a fixed real number $\lambda>1$. See also $[1,12,13]$ for constructions and more properties of pseudo-Anosov maps. By the Nielsen-Thurston classification of surface homeomorphisms [5, 14], a nonperiodic map $f$ is either isotopic to a reducible map, or isotopic to a pseudo-Anosov map.

The simplest nontrivial reducible map is the Dehn twist $t_{\alpha}$ along a simple closed geodesic $\alpha$ that is obtained by cutting $S$ along $\alpha$, rotating one of the copies of $\alpha$ by 360 degrees and then gluing the two copies back together. The problem of determining whether a finite product of a pseudo-Anosov map $f$ and a power of $t_{\alpha}$ is still isotopic to a pseudo-Anosov map was extensively studied in $[4,8,9]$. By an abuse of language we call a map $f$ pseudo-Anosov if $f$ is isotopic to a pseudo-Anosov map, and we call a mapping class $\theta$ pseudo-Anosov if one of its representatives is a pseudo-Anosov map. In [9] Long and Morton proved that $t_{\alpha}^{k} \circ f$ are pseudo-Anosov for all but at most

(C) 2010 Australian Mathematical Publishing Association Inc. 1446-7887/2010 \$16.00 
a finite number of integer values of $k$. Later, Fathi [4] found that $t_{\alpha}^{k} \circ f$ are pseudoAnosov for all but at most seven consecutive integer values of $k$. In [3] Boyer et al. improved the number 'seven' to 'six'.

The main purpose of this paper is to identify certain classes of pseudo-Anosov maps $f$ on a Riemann surface $S$ with punctures so that $t_{\alpha}^{k} \circ f$ are pseudo-Anosov for all integers $k$. Throughout the paper we assume that $S$ is of type $(p, n)$ with $3 p+n>4$ and $n \geq 1$. Let $a$ denote a puncture of $S$. Write $\tilde{S}=S \cup\{a\}$. Let $\operatorname{Mod}_{S}^{a}$ be the group that consists of isotopy classes of self-maps of $S$ fixing the puncture $a$. Each element of

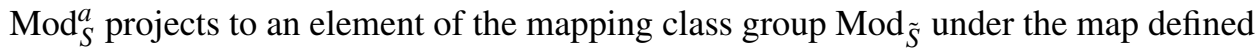
by neglecting the puncture $a$, thus defining a natural group epimorphism

$$
i: \operatorname{Mod}_{S}^{a} \rightarrow \operatorname{Mod}_{\tilde{S}}
$$

Let $\mathbf{H}$ be the hyperbolic plane, and $\varrho: \mathbf{H} \rightarrow \tilde{S}$ the universal covering with a group $G$ of deck transformations. Since $\tilde{S}$ is a compact surface with a finite number of points removed, the group $G$ is finitely generated, torsion free, and of the first kind. Following Bers [2], there is a fiber space $F(\tilde{S})$, referred to in the literature as a Bers fiber space, over the Teichmüller space $T(\tilde{S})$ such that $F(\tilde{S})$ is isomorphic (via an isomorphism $\varphi$ ) to the Teichmüller space $T(S)$. Furthermore, $\operatorname{Mod}_{\tilde{S}}$ extends to a $\operatorname{group} \bmod (\tilde{S})$ of fiber-preserving holomorphic automorphisms of $F(\tilde{S})$ and the isomorphism $\varphi$ induces a group isomorphism $\varphi^{*}: \bmod (\tilde{S}) \rightarrow \operatorname{Mod}_{S}^{a}$. Since $G$ can be regarded as a normal subgroup of $\bmod (\tilde{S})$, the $\operatorname{group} \varphi^{*}(G)$ is a normal subgroup of $\operatorname{Mod}_{S}^{a}$ that consists of mapping classes $\theta$ with $i(\theta)=\mathrm{id}$.

By [11, Theorem 2] or [7, Theorem 2], for any primitive simple hyperbolic element $h \in G$, its $\varphi^{*}$-image $\varphi^{*}(h)$ is represented by a spin $t_{\alpha_{1}}^{-1} \circ t_{\alpha_{0}}$, where the pair $\left\{\alpha_{1}, \alpha_{0}\right\}$ bounds an $a$-punctured cylinder on $S$. Denote

$$
\mathcal{F}(S, a)=\left\{\theta \in \operatorname{Mod}_{S}^{a}: \theta \text { is pseudo-Anosov and } i(\theta)=\mathrm{id}\right\} .
$$

By [7, Theorem 2], elements of $\mathcal{F}(S, a)$ are $\varphi^{*}$-images of essential hyperbolic elements $g$ of $G$, where $g$ is called essential if its axis $c_{g}$ projects to a geodesic $\varrho\left(c_{g}\right)$ on $\tilde{S}$ that intersects every simple closed geodesic on $\tilde{S}$. For each simple closed geodesic $\tilde{\alpha}$ on $\tilde{S}$, let $t_{\tilde{\alpha}}$ denote the Dehn twist along $\tilde{\alpha}$. Let $\hat{\alpha}$ be a geodesic in $\mathbf{H}$ with $\varrho(\hat{\alpha})=\tilde{\alpha}$. Let $U_{\hat{\alpha}}$ be a component of $\mathbf{H}-\{\hat{\alpha}\}$. Associated with $U_{\hat{\alpha}}$ there is a lift $\tau_{\hat{\alpha}}: \mathbf{H} \rightarrow \mathbf{H}$ of $t_{\tilde{\alpha}}$ that in turn determines a disjoint union of half-planes $U_{i}$, $i=1,2, \ldots$, so that the region

$$
\Sigma_{\hat{\alpha}}=\mathbf{H}-\bigcup U_{i} \subset \mathbf{H}-U_{\hat{\alpha}}
$$

is not empty and $\left.\tau_{\hat{\alpha}}\right|_{\Sigma_{\hat{\alpha}}}=\mathrm{id}$. We call those half-planes $U_{i}$ maximal elements of $\tau_{\hat{\alpha}}$.

The lift $\tau_{\hat{\alpha}}$ defines an element $\left[\tau_{\hat{\alpha}}\right]$ of $\bmod (\tilde{S})$. By [15, Lemma 3.2], we assert that $\varphi^{*}\left(\left[\tau_{\hat{\alpha}}\right]\right) \in \operatorname{Mod}_{S}^{a}$ is represented by the Dehn twist $t_{\alpha}$ along a simple closed geodesic $\alpha$ on $S$ that is freely homotopic to $\tilde{\alpha}$ on $\tilde{S}$ (as $a$ is filled in). The main result of this paper is the following. 
THEOREM 1.1. Let $g \in G$ be an essential hyperbolic element, and let $c_{g}$ be the axis of $g$. Assume that $c_{g} \cap \Sigma_{\hat{\alpha}} \neq \emptyset$. Then for any integer $k$, the mapping class $t_{\alpha}^{k} \circ \varphi^{*}(g)$ is pseudo-Anosov.

REMARK. Theorem 1.1 is no longer valid if we drop the assumption that $c_{g} \cap \Sigma_{\alpha} \neq \emptyset$. In Section 8, we show that for any geodesic $\alpha \subset S$, there exists an essential hyperbolic element $g \in G$ with $c_{g} \cap \Sigma_{\hat{\alpha}}=\emptyset$ such that the product $t_{\alpha}^{k} \circ \varphi^{*}(g)$ fails to be pseudoAnosov for some integer $k$.

From Theorem 1.1, we can prove for certain reducible mapping classes $\theta \in \operatorname{Mod}_{S}^{a}$ that there exists a simple closed geodesic $\alpha$ on $S$ such that $t_{\alpha} \circ \theta$ is pseudo-Anosov. To state our next result, we call an element $h \in G$ a simple hyperbolic if it is hyperbolic and its axis $c_{h}$ projects to a simple closed geodesic $\varrho\left(c_{h}\right)$ on $\tilde{S}$. We will prove the following corollary.

COROLlaRY 1.2. Let $h, g \in G$ be hyperbolic elements such that $h$ is simple and $h g$ is essential. Write $\varphi^{*}(h)=t_{\alpha}^{-1} \circ t_{\alpha_{0}}$. If $c_{g}$ intersects $c_{h}$, then the mapping classes $t_{\alpha}^{-1} \circ \varphi^{*}(g)$ and $t_{\alpha_{0}} \circ \varphi^{*}(g)$ are both pseudo-Anosov.

Since for every essential hyperbolic element $g \in G$ there is an essential hyperbolic element $g_{0}$ in its conjugacy class in $G$ such that the axis $c_{g_{0}}$ of $g_{0}$ meets $\Sigma_{\hat{\alpha}}$, Theorem 1.1 has another immediate corollary.

COROLlary 1.3. For any essential hyperbolic element $g \in G$ and any simple closed geodesic $\alpha \subset S$ that is nontrivial on $\tilde{S}$, there is an element $e \in G$ such that for any integer $k$, the mapping class $t_{\alpha}^{k} \circ \varphi^{*}\left(e g e^{-1}\right)$ is pseudo-Anosov.

The argument of Theorem 1.1 can be used to prove that any finite product

$$
\prod_{i}\left(t_{\alpha}^{k_{i}} \circ f\right)
$$

where $k_{i} \in \mathbf{Z}$, is pseudo-Anosov for $f=\varphi^{*}(g) \in \mathcal{F}(S, a)$ provided that the axis of $g$ meets $\Sigma_{\hat{\alpha}}$.

Now we briefly discuss a generalization of Theorem 1.1 that is related to a problem posed in [6]. By Fathi's result [4], for any geodesic $\alpha$ and any pseudo-Anosov map $f$ of $S$, the products $t_{\alpha}^{k} \circ f$ are pseudo-Anosov for all but at most seven consecutive integers. The question asks if it is possible to replace $t_{\alpha}^{k}$ by a multi-twist $\prod_{i} t_{\alpha_{i}}^{k_{i}}$ for an integer tuple $\left(k_{1}, \ldots k_{m}\right)$ and a collection $\left(\alpha_{1}, \ldots, \alpha_{m}\right)$ of $m$ disjoint and independent simple closed geodesics on $S$, where $1 \leq m \leq 3 p-3+n$, possibly at the cost of replacing the number seven by an undetermined but universal number $N$. Let

$$
\left\{\tilde{\alpha}_{1}, \ldots, \tilde{\alpha}_{m}\right\} \subset \tilde{S}, \quad 1 \leq m \leq 3 p-4+n,
$$

be a collection of disjoint simple closed geodesics, and let $\left[\tau_{i}\right] \in \bmod (\tilde{S}), 1 \leq i \leq m$, be the lifts of $t_{\tilde{\alpha}_{i}}$ such that $\varphi^{*}\left(\left[\tau_{i}\right]\right)=t_{\alpha_{i}}$. Then $\alpha_{1}, \ldots, \alpha_{m}$ may not be disjoint. But if the region

$$
\Sigma_{0}=\mathbf{H}-\left\{\text { all maximal elements of } \tau_{1}, \tau_{2}, \ldots, \tau_{m}\right\}
$$


is not empty, then $\alpha_{1}, \ldots, \alpha_{m}$ are mutually disjoint. Again, the methods of Theorem 1.1 can be employed to conclude that for any essential element $g \in G$ with $c_{g} \cap \Sigma_{0} \neq \emptyset$, the mapping class

$$
\left(\prod_{i} t_{\alpha_{i}}^{k_{i}}\right) \circ f
$$

where $k_{i} \in \mathbf{Z}$, is pseudo-Anosov for $f=\varphi^{*}(g) \in \mathcal{F}(S, a)$.

This paper is organized as follows. Section 2 is background material that is needed in the proof of Theorem 1.1. In Section 3, we study those reducible mapping classes on $S$ isotopic to a Dehn twist on $\tilde{S}$, and investigate the properties of the corresponding curve systems. In Section 4, we interpret those reducible mapping classes as elements of $\bmod (\tilde{S})$, and study their actions on $\mathbf{S}^{1}$. In Section 5 , we prove that if $\theta=t_{\alpha}^{k} \circ f$, where $f \in \mathcal{F}(S, a)$, is reducible by a curve system $\left\{c_{1}, \ldots, c_{s}\right\}$, then all $c_{i}$ are nontrivial on $\tilde{S}$. In Section 6, we prove Theorem 1.1. Section 7 is devoted to the proof of Corollary 1.2. In Section 8, we present an example to illustrate that Theorem 1.1 will not be true if the condition that $c_{g} \cap \Sigma_{\hat{\alpha}} \neq \emptyset$ is dropped.

\section{Preliminaries}

We fix a Riemann surface $\tilde{S}$ as introduced above, and consider all possible pairs $\left(\tilde{S}_{1}, w_{1}\right)$ where $\tilde{S}_{1}$ is a Riemann surface of the same type $(p, n)$ and $w_{1}: \tilde{S} \rightarrow \tilde{S}_{1}$ is a quasiconformal homeomorphism. Two pairs $\left(\tilde{S}_{1}, w_{1}\right)$ and $\left(\tilde{S}_{2}, w_{2}\right)$ are equivalent if the map $w_{2} \circ w_{1}^{-1}: \tilde{S}_{1} \rightarrow \tilde{S}_{2}$ is isotopic to a conformal map. The Teichmüller space $T(\tilde{S})$ is defined as the set of pairs $\left(\tilde{S}_{1}, w_{1}\right)$ modulo the equivalence relation. Note that every pair $\left(\tilde{S}_{1}, w_{1}\right)$ defines a new conformal structure $\mu_{1}$ on $\tilde{S}$ via pullbacks. Two conformal structures $\mu_{1}$ and $\mu_{2}$ are equivalent if $\left(\tilde{S}_{1}, w_{1}\right)$ is equivalent to $\left(\tilde{S}_{2}, w_{2}\right)$. Denote by $[\mu]$ the equivalence class of $\mu$.

Associated with each point $[\mu] \in T(\tilde{S})$ there is a Jordan domain $w^{\mu}(\mathbf{H})$ depending holomorphically on $[\mu]$, where $w^{\mu}: \mathbf{C} \rightarrow \mathbf{C}$ is a quasiconformal map that satisfies $w^{\mu}(0)=0, w^{\mu}(1)=1, w^{\mu}$ is conformal off $\mathbf{H}$, and $\partial_{z} w^{\mu}(z) / \partial_{\bar{z}} w^{\mu}(z)=\mu(z)$ for all $z \in \mathbf{H}$. We then form the Bers fiber space

$$
F(\tilde{S})=\left\{([\mu], z):[\mu] \in T(\tilde{S}) \text { and } z \in w^{\mu}(\mathbf{H})\right\} .
$$

The Bers isomorphism theorem [2, Theorem 9] states that there is an isomorphism $\varphi: F(\tilde{S}) \rightarrow T(S)$.

By definition, $\operatorname{Mod}_{\tilde{S}}$ consists of isotopy classes of self-maps of $\tilde{S}$. Let $\zeta \in \operatorname{Mod}_{\tilde{S}}$ be induced by a self-map $w$ of $\tilde{S}$. The map $w$ can be lifted to an automorphism $\omega: \mathbf{H} \rightarrow \mathbf{H}$ under the universal covering $\varrho: \mathbf{H} \rightarrow \tilde{S}$. We call $\omega, \omega^{\prime}: \mathbf{H} \rightarrow \mathbf{H}$ equivalent if $\omega g \omega^{-1}=\omega^{\prime} g \omega^{\prime-1}$ for every element $g \in G$. The equivalence class of $\omega$ is denoted by $[\omega]$. The $\operatorname{group} \bmod (\tilde{S})$ is a collection of $[\omega]$ for all maps $w: \tilde{S} \rightarrow \tilde{S}$. The Bers isomorphism $\varphi: F(\tilde{S}) \rightarrow T(S)$ induces an isomorphism $\varphi^{*}: \bmod (\tilde{S}) \rightarrow \operatorname{Mod}_{S}^{a}$ defined by conjugation. Since the covering group $G$ is regarded as a normal subgroup 
of $\bmod (\tilde{S})$, the image group $\varphi^{*}(G)$ is a normal subgroup of $\operatorname{Mod}_{S}^{a}$ consisting of elements $\theta$ such that $i(\theta)=$ id. Let $[\omega] \in \bmod (\tilde{S})$ be such that $\varphi^{*}([\omega])=\theta$. Then $[\omega] \in G$. There are three cases to consider.

Case 1. $[\omega] \in G$ is a simple hyperbolic element. Let $c$ be its axis and write $\tilde{c}=\varrho(c)$. By [11, Theorem 2] or [7, Theorem 2], we see that $\varphi^{*}([\omega])$ can be represented in the form $t_{\alpha}^{-k} \circ t_{\alpha_{0}}^{k}$ for an integer $k$, where $\left\{\alpha, \alpha_{0}\right\}$ bounds an $a$-punctured cylinder on $S$. Both $\alpha$ and $\alpha_{0}$ are simple closed geodesics homotopic to $\tilde{c}$ on $\tilde{S}$ as $a$ is filled in.

Case 2. $[\omega] \in G$ is parabolic. In this case, the mapping class $\varphi^{*}([\omega])$ is represented by an ordinary power of a Dehn twist along a curve $c$, where $c$ bounds a twice punctured disk on $S$ enclosing the puncture $a$ and another puncture of $\tilde{S}$ corresponding to the conjugacy class of the parabolic element.

Case 3. $[\omega] \in G$ is essential hyperbolic. In this case, the mapping class $\varphi^{*}([\omega])$ is pseudo-Anosov.

We proceed to investigate some special elements $[\omega]$ in $\bmod (\tilde{S})-G$ as well as its image in $\operatorname{Mod}_{S}^{a}$ under the Bers isomorphism $\varphi^{*}: \bmod (\tilde{S}) \rightarrow \operatorname{Mod}_{S}^{a}$. Let $\tilde{\alpha}$ be a simple closed geodesic on $\tilde{S}$ and $\hat{\alpha} \in \mathbf{H}$ a geodesic such that $\tilde{\alpha}=\varrho(\hat{\alpha})$. Let $U$ and $U^{*}$ be the components of $\mathbf{H}-\hat{\alpha}$. As mentioned earlier, the Dehn twist $t_{\tilde{\alpha}}$ can be lifted to a quasiconformal homeomorphism $\tau: \mathbf{H} \rightarrow \mathbf{H}$ with respect to $U$ in the following way. Let $h \in G$ be a primitive simple hyperbolic element such that $h(U)=U$. We take an earthquake $h$-shift on $U$ and leave $U^{*}$ fixed. We then define $\tau$ via $G$-invariance.

Obviously, the map $\tau$ gives rise to a collection $\mathcal{U}$ of layered half-planes in $\mathbf{H}$ in a partial order defined by inclusion. There are infinitely many disjoint maximal elements of $\mathcal{U}$ such that the complement $\Sigma$ of all maximal elements in $\mathbf{H}$ is nonempty and simply connected. The map $\tau$ keeps each maximal element of $\mathcal{U}$ invariant and the restriction $\left.\tau\right|_{\Sigma}$ is the identity.

Let $\mathbf{Q} \subset \mathbf{S}^{1}$ denote the dense subset consisting of points covered by finitely many elements of $\mathcal{U}$. Choose $z \in \mathbf{Q}$ and let $U=U_{0} \supset U_{1} \supset \cdots \supset U_{m}, U_{i} \in \mathcal{U}$, cover $z$. Let $h_{i}, i=0,1, \ldots m$, denote the primitive simple hyperbolic elements of $G$ that keep $U_{i}$ invariant and take the same orientation as $h_{0}=h$. Then $\tau(z)$ is defined as

$$
\tau(z)=h_{0} h_{1} \cdots h_{m}(z) .
$$

For a point $z \in \mathbf{S}^{1}$ not covered by any elements of $\mathcal{U}$, we have $\tau_{i}(z)=z$. Now for any other point $z \in \mathbf{S}^{1}-\mathbf{Q}$, we choose a sequence $\left\{z_{j}\right\} \subset \mathbf{Q}$ with $z_{j} \rightarrow z$. We have $\tau(z)=\lim _{j \rightarrow \infty} \tau\left(z_{j}\right)$.

The equivalence class of $\tau$ determines an element $[\tau]$ of $\bmod (\tilde{S})$. By $[15$, Lemma 3.3], the mapping class $\varphi^{*}([\tau])$ is represented by a Dehn twist $t_{\alpha}$ for a simple closed geodesic $\alpha \subset S$. For this reason, in the rest of this paper we use the symbols $\tau_{\alpha}, \mathcal{U}_{\alpha}$ and $\Sigma_{\alpha}$ to denote $\tau, \mathcal{U}$ and $\Sigma$, respectively. Observe that if $\tau_{\alpha}$ is a lift of $t_{\tilde{\alpha}}$ with respect to $U$, then $h^{-1} \tau_{\alpha}$ is also a lift of $t_{\tilde{\alpha}}$ with respect to $U^{*}$. But $\varphi^{*}\left(\left[h^{-1} \tau_{\alpha}\right]\right)$ is represented by $t_{\alpha_{0}}$, where $\alpha_{0}$ together with $\alpha$ bounds an $a$-punctured cylinder on $S$. 
By [15, Lemma 3.3] again, for every simple closed geodesic $\alpha \subset S$, there exists a lift $\tau_{\alpha}$ of $t_{\tilde{\alpha}}$ such that $\varphi^{*}\left(\left[\tau_{\alpha}\right]\right)=t_{\alpha}$.

Let $[\omega] \in \bmod (\tilde{S})-G$ be a lift of $t_{\tilde{\alpha}}$. Then $[\omega]$ is of the form $\left[\tau_{\alpha}\right] h$ for some $h \in G$. In this case, the mapping class $\varphi^{*}([\omega])$ is the product of $t_{\alpha}$ and $\varphi^{*}(h)$. Suppose that $G$ has a parabolic fixed point $x$, and that $T \in G$ is the parabolic element so that $T(x)=x$. By [15, Lemma 3.1], we have $x \in \mathbf{Q}$. Hence there are only finitely many elements of $\mathcal{U}_{\alpha}$ that cover $x$. It follows that every parabolic fixed point $x$ of $G$ is associated with a positive integer $\epsilon\left(\tau_{\alpha}, x\right)$ that is the number of elements of $\mathcal{U}_{\alpha}$ containing $x$. It is evident that $\epsilon\left(\tau_{\alpha}, x\right)=\epsilon\left(\tau_{\alpha}, \tau_{\alpha}(x)\right) \neq 0$ if $x$ is covered by a maximal element of $\mathcal{U}_{\alpha}$, and $\epsilon\left(\tau_{\alpha}, x\right)=0$ if and only if $x$ lies outside of all maximal elements of $\mathcal{U}_{\alpha}$. In the latter case, the parabolic element $T$ commutes with $\tau_{\alpha}$ and the geodesic $\alpha$ on $S$ determined by $t_{\alpha}=\varphi^{*}\left(\left[\tau_{\alpha}\right]\right)$ is disjoint from the boundary of the twice punctured disk determined by $\varphi^{*}(T)$ [11, Theorem 2].

\section{Reducible mapping classes and curves}

Let $\theta \in \mathcal{F}(S, a)$. Let $g \in G$ be such that $\varphi^{*}(g)=\theta$. Let $\alpha \subset S$ be a simple closed geodesic so that $\alpha$ is also nontrivial on $\tilde{S}$. Then $\alpha$ is not a geodesic on $\tilde{S}$ when $a$ is filled in. In what follows, we use $\tilde{\alpha}$ to denote the geodesic homotopic to $\alpha$ on $\tilde{S}$. Assume that $\theta=t_{\alpha}^{k} \circ \varphi^{*}(g)$ is not pseudo-Anosov. Then there is a system

$$
\mathcal{C}=\left\{c_{1}, \ldots, c_{s}\right\}
$$

where $s \geq 1$, of disjoint simple closed geodesics on $S$ that is invariant under a suitable representative of $\theta$. We assume that every curve in $\mathcal{C}$ is also nontrivial on $\tilde{S}$. The case in which $\mathcal{C}$ contains a curve $c$ that is trivial on $\tilde{S}$ will be handled in Section 5. We can write

$$
\theta(\mathcal{C})=\mathcal{C}
$$

Let $\Lambda$ be the set of simple closed geodesics $c$ on $S$ that project to nontrivial simple closed geodesics $\tilde{c}$ so that $\tilde{c}=\tilde{\alpha}$ or $\tilde{c}$ is disjoint from $\tilde{\alpha}$. Let $\Lambda_{1}$ be the subset of $\Lambda$ consisting of geodesics $c$ such that near the puncture $a$, the geodesics $c$ and $\alpha$ bound a bigon $B$ enclosing $a$. Let $\Lambda_{2}=\Lambda-\Lambda_{1}$. Then $\Lambda_{1} \cup \Lambda_{2}=\Lambda$ and $\Lambda_{2}$ consist of geodesics $c$ on $S$ that are nontrivial on $\tilde{S}$ and are equal to or disjoint from $\alpha$.

LeMma 3.1. $\mathcal{C} \subset \Lambda$.

PROOF. By taking a suitable power of $c_{i}$, we may assume that $\theta\left(c_{i}\right)=c_{i}$ for every $i=1, \ldots, s$. Assume that there is a $c_{1} \in \mathcal{C}$, say, so that $\tilde{c}_{1} \subset \tilde{S}$ is nontrivial and intersects $\tilde{\alpha}$.

Since $i(\theta)=t_{\tilde{\alpha}}^{k}$, the Dehn twist $t_{\tilde{\alpha}}^{k}$ keeps $\tilde{c}_{1}$ invariant. By hypothesis, the curve $\tilde{c}_{1} \subset \tilde{S}$ intersects $\tilde{\alpha}$, which means that the image loop $t_{\tilde{\alpha}}^{k}\left(\tilde{c}_{1}\right)$ intersects $\tilde{c}_{1}$. It follows that $t_{\tilde{\alpha}}^{k}$ sends $\tilde{c}_{1}$ to a different homotopy class. This is a contradiction.

Note that $\theta$ may not keep each element of $\mathcal{C}$ invariant. Let $\mathcal{C}_{0}$ be the subset of $\mathcal{C}$ consisting of curves in $\mathcal{C}$ with $\theta(c) \neq c$. 
LEMMA 3.2. $\mathcal{C}_{0}$ contains at most two curves. In other words, the mapping class $\theta^{2}$ keeps each element of $\mathcal{C}$ invariant. Further, if $\left\{c_{1}, c_{2}\right\}=\mathcal{C}_{0}$, then $\left\{c_{1}, c_{2}\right\}$ bounds an a-punctured cylinder on $S$.

PROOF. Suppose that $\mathcal{C}_{0}$ consists of at least three curves $c_{1}, c_{2}$ and $c_{3}$. Since there are at most two disjoint curves $c$ and $c^{\prime}$ on $S$ so that $\tilde{c}=\tilde{c}^{\prime}$, we may assume that $\theta\left(c_{1}\right)=c_{2}$ and $\left\{c_{1}, c_{2}\right\}$ does not bound an $a$-punctured cylinder on $S$. That is, the geodesic $\tilde{c}_{1}$ is disjoint from $\tilde{c}_{2}$. Since $\theta\left(c_{1}\right)=c_{2}$, by filling in the puncture $a$, we obtain

$$
i(\theta)\left(\tilde{c}_{1}\right)=\tilde{c}_{2} .
$$

On the other hand, we recall that $i(\theta)=t_{\tilde{\alpha}}$. From Lemma 3.1, $\mathcal{C}_{0} \subset \Lambda$. We see that both $\tilde{c}_{1}$ and $\tilde{c}_{2}$ are disjoint from $\tilde{\alpha}$. This implies that $t_{\tilde{\alpha}}\left(\tilde{c}_{1}\right)=\tilde{c}_{1}$ and $t_{\tilde{\alpha}}\left(\tilde{c}_{2}\right)=\tilde{c}_{2}$. This contradicts (3.2).

Lemma 3.3. Suppose $\mathcal{C} \cap \Lambda_{1}$ is empty. Then $\theta$ is not reduced by the system $\mathcal{C}$.

PROOF. By Lemma 3.1, we have $\mathcal{C} \subset \Lambda$. So if $\mathcal{C} \cap \Lambda_{1}$ is empty, then every curve in $\mathcal{C}$ must be in $\Lambda_{2}$. Therefore $t_{\alpha}$ commutes with $t_{j}$ for $1 \leq j \leq s$, where for simplicity $t_{j}=t_{c_{j}}$. Suppose that $\theta=t_{\alpha}^{k} \circ \varphi^{*}(g)$ is reduced by $\mathcal{C}$. Then

$$
\left(t_{1} \circ \cdots \circ t_{s}\right) \circ\left(t_{\alpha}^{k} \circ \varphi^{*}(g)\right)=\left(t_{\alpha}^{k} \circ \varphi^{*}(g)\right) \circ\left(t_{1} \circ \cdots \circ t_{s}\right) .
$$

Since $t_{\alpha}$ commutes with each $t_{j}$ for $1 \leq j \leq s$, we obtain

$$
\left(t_{1} \circ \cdots \circ t_{s}\right) \circ \varphi^{*}(g)=\varphi^{*}(g) \circ\left(t_{1} \circ \cdots \circ t_{s}\right) .
$$

Recall that $g \in G$ is essential. We see that (3.3) cannot hold since it says that $\varphi^{*}(g)$ keeps $c_{1}, \ldots, c_{s}$ invariant.

It follows from Lemma 3.3 that $\mathcal{C} \cap \Lambda_{1} \neq \emptyset$. Consequently, we can choose a curve $c \in \mathcal{C} \cap \Lambda_{1}$. By Lemma 3.2, we can take a square of $\theta$ if necessary, and may assume that $\theta(c)=c$. Let $\tau_{c}: \mathbf{H} \rightarrow \mathbf{H}$ be the lift of $t_{\tilde{c}}$ so that $\varphi^{*}\left(\left[\tau_{c}\right]\right)$ is represented by $t_{c}$. We have the following result.

LEMMA 3.4. The pair $\left(\tau_{\alpha}, \tau_{c}\right)$ satisfies the following properties.

(1) The geodesic boundary $\partial W_{0}$ of any maximal element $W_{0}$ of $\mathcal{U}_{c}$ is disjoint from the geodesic boundary $\partial U_{0}$ of any maximal element $U_{0}$ of $\mathcal{U}_{\alpha}$.

(2) There exist maximal elements $U$ and $W$ of $\mathcal{U}_{\alpha}$ and $\mathcal{U}_{c}$, respectively, such that $U \cap W \neq \emptyset$ and $U \cup W=\mathbf{H}$.

(3) For any maximal element $U_{0} \neq U$ of $\mathcal{U}_{\alpha}$, we have $U_{0} \subset W$.

PROOF. Since $c \in \mathcal{C} \cap \Lambda_{1} \subset \Lambda$, the geodesic $\tilde{c}$ is disjoint from $\tilde{\alpha}$. So every geodesic in the set $\left\{\varrho^{-1}(\tilde{c})\right\}$ of preimages of $\tilde{c}$ is disjoint from any geodesic in the set $\left\{\varrho^{-1}(\tilde{\alpha})\right\}$ of preimages of $\tilde{\alpha}$. But the geodesic boundary $\partial U$ is one of the elements in $\left\{\varrho^{-1}(\tilde{\alpha})\right\}$ and $\partial W$ is one of the elements in $\left\{\varrho^{-1}(\tilde{c})\right\}$. This proves (1). 
To prove (2) of the lemma, we suppose that there is no such pair $(U, W)$. That is, for any maximal element $U \in \mathcal{U}_{\alpha}$ and any maximal element $W \in \mathcal{U}_{c}$, either $U \subset W$, or $W \subset U$, or $U, W$ are disjoint. Suppose that $U \subset W$. For any hyperbolic element $h \in G$ whose repelling fixed point is contained in $U \cap \mathbf{S}^{1}$ and whose attracting fixed point lies outside of $W$, by construction, the region $h(\mathbf{H}-U)$ is contained in a maximal element $U^{\prime}$ of $\mathcal{U}_{\alpha}$. By assumption, the half-plane $U^{\prime}$ is disjoint from $W$. It follows that $\Sigma_{\alpha} \cap \Sigma_{c} \neq \emptyset$ (where $\Sigma_{\alpha}$ and $\Sigma_{c}$ are defined as in (2)) and the boundary components of $\Sigma_{\alpha} \cap \Sigma_{c}$ are either $\partial U$ for some $U \in \mathcal{U}_{\alpha}$, or $\partial W$ for some $W \in \mathcal{U}_{c}$. This implies that $\left[\tau_{c}\right]$ commutes with $\left[\tau_{\alpha}\right]$. Via the Bers isomorphism $\varphi^{*}: \bmod (\tilde{S}) \rightarrow \operatorname{Mod}_{S}^{a}$, we see that $t_{c}$ commutes with $t_{\alpha}$. This implies that $c$ and $\alpha$ are disjoint, which contradicts the fact that $c \in \mathcal{C} \cap \Lambda_{1}$.

(3) is obvious. If $U_{0} \neq U$, then $U_{0}$ and $U$ are disjoint. Thus $U_{0} \subset \mathbf{H}-U$. From (2), we have $U_{0} \subset W$. Lemma 3.4 is proved.

Suppose that $c_{g}$ intersects $\Sigma_{\alpha}$. Then there is a maximal element $U \in \mathcal{U}_{\alpha}$ such that $c_{g}$ intersects $\partial U$. Also assume that $U$ contains the repelling (but not attracting) fixed point of $g$. Let $U_{0} \in \mathcal{U}_{\alpha}$ be another maximal element that contains $g(\mathbf{H}-U)$. Then $U_{0}$ must be disjoint from $U$. Under the circumstances, a slight modification of the argument of Lemma 3.4 leads to the following result.

LEMMA 3.5. Let $U, U_{0} \in \mathcal{U}_{\alpha}$ be maximal elements defined as above. Then there exists a maximal element $W \in \mathcal{U}_{c}$ such that either one of $(U, W)$ and $\left(U_{0}, W\right)$ satisfies condition (2) of Lemma 3.4, or both $U$ and $U_{0}$ are contained in $W$.

Proof. Choose a maximal element $W \in \mathcal{U}_{c}$ so that $W$ is not disjoint from $U$. If ( $U, W$ ) satisfies condition (2) of Lemma 3.4, we are done. Otherwise, either $U \subset W$, or $W \subset U$.

Suppose that $U \subset W$. Let $U_{0} \in \mathcal{U}_{\alpha}$ be a maximal element that includes $g(\mathbf{H}-U)$. Assume that $U_{0}$ is not contained in $W$. If $\left(U_{0}, W\right)$ satisfies condition (2) of Lemma 3.4, we are done. Otherwise, we see that $\Sigma_{\alpha} \cap \Sigma_{c} \neq \emptyset$. Now the argument of Lemma 3.4(2) can be applied to show that $\tau_{c}$ commutes with $\tau_{\alpha}$. But this would contradict the fact that $c \in \mathcal{C} \cap \Lambda_{1}$.

If $W \subset U$, then we consider the set $\mathcal{M}$ of all maximal elements of $\mathcal{U}_{c}$ that contains $h(\mathbf{H}-W)$, where $h$ runs over all hyperbolic elements whose attracting fixed point lies outside of $W$ and whose repelling fixed point lies in $W \cap \mathbf{S}^{1}$. If $\mathcal{M}$ contains an element $W^{\prime}$ such that $\left(U, W^{\prime}\right)$ satisfies condition (2) of Lemma 3.4, we are done. Otherwise, the map $\tau_{c}$ commutes with $\tau_{\alpha}$, in contradiction to $c \in \mathcal{C} \cap \Lambda_{1}$.

\section{Reducible mapping classes interpreted as elements of $\bmod (\tilde{S})$}

In this section we discuss certain reducible mapping classes by virtue of elements of $\bmod (\tilde{S})$. Let $c \subset S$ be a simple closed geodesic. Let $\chi \in \operatorname{Mod}_{S}^{a}$ be a reducible mapping class by a curve system containing $c$. Let $[\omega] \in \bmod (\tilde{S})$ be an element such that $\varphi^{*}([\omega])=\chi$. 
LEMMA 4.1. Suppose that $\tilde{c}$ is nontrivial and that $\chi(c)=c$. Let $\left[\tau_{c}\right] \in \bmod (\tilde{S})$ be the element such that $\varphi^{*}\left(\left[\tau_{c}\right]\right)$ is represented by $t_{c}$. Then there exists a map in the equivalence class $[\omega]$ (which is denoted by $\omega$ also) such that $\omega$ keeps the set of maximal elements of $\mathcal{U}_{c}$ invariant.

PROOF. By assumption, we may choose a representative $\sigma$ for $\chi$ so that $\sigma(c)=c$. It is then obvious that $\sigma$ commutes with the Dehn twist $t_{c}$. That is,

$$
\sigma \circ t_{c} \circ \sigma^{-1}=t_{c}
$$

From (4.1) and the Bers isomorphism $\varphi^{*}$, we obtain

$$
[\omega]\left[\tau_{c}\right][\omega]^{-1}=\left[\tau_{c}\right]
$$

Let $W$ be any maximal element of $\mathcal{U}_{c}$. Choose a representative $\omega^{\prime}$ of $[\omega]$. Obviously, the map $\tau_{c}$ keeps $W \cap \mathbf{S}^{1}$ invariant, and no points in the interior of $W \cap \mathbf{S}^{1}$ in $\mathbf{S}^{1}$ are fixed by $\tau_{c}$. Then $\left.\omega^{\prime} \tau_{c} \omega^{\prime-1}\right|_{\mathbf{S}^{1}}$ sends $\omega^{\prime}(W) \cap \mathbf{S}^{1}$ to itself and does not fix any point in $\omega^{\prime}(W) \cap \mathbf{S}^{1}$. From (4.2), we see that $\left.\tau_{c}\right|_{\mathbf{S}^{1}}$ sends $\omega^{\prime}(W) \cap \mathbf{S}^{1}$ to itself and does not fix any point in $\omega^{\prime}(W) \cap \mathbf{S}^{1}$. This implies that there is a representative $\omega$ of $[\omega]$ such that $\omega(W)$ is also a maximal element of $\mathcal{U}_{c}$.

The following result was proved in [16].

LEMMA 4.2. If $\tilde{c}$ is trivial, then $\chi(c)=c$ and every representative $\omega$ of $[\omega]$ fixes a parabolic fixed point of $G$.

For every maximal element $W \in \mathcal{U}_{c}$, we write $\bar{W}=W \cup \partial W$ and $W^{*}=\mathbf{H}-W$. Also, the complement of an $\operatorname{arc} \Gamma$ in $\mathbf{S}^{1}$ is denoted by $\Gamma^{c}$.

LEMMA 4.3. Let $W$ be a maximal element of $\mathcal{U}_{c}$. Let $[\omega] \in \bmod (\tilde{S})$. If the intersection $\omega\left(W^{*}\right) \cap W^{*}=\emptyset$ for a representative $\omega$ of $[\omega]$, then for any representative $\omega_{0}$ of $[\omega]$, the region $\omega_{0}(W)$ is not a maximal element of $\mathcal{U}_{c}$.

PROOF. First, observe that $\omega$ is a quasiconformal homeomorphism of $\mathbf{H}$. For any representative $\omega_{0}$ of $[\omega]$ we must have $\left.\omega\right|_{\mathbf{S}^{1}}=\left.\omega_{0}\right|_{\mathbf{S}^{1}}$. The hypothesis implies that $\left(\omega_{0}\left(W^{*}\right) \cap \mathbf{S}^{1}\right) \cap\left(W^{*} \cap \mathbf{S}^{1}\right)=\emptyset$. It follows that

$$
\begin{aligned}
\mathbf{S}^{1} & =\left(\left(\omega_{0}\left(W^{*}\right) \cap \mathbf{S}^{1}\right) \cap\left(W^{*} \cap \mathbf{S}^{1}\right)\right)^{c}=\left(\omega_{0}\left(W^{*}\right) \cap \mathbf{S}^{1}\right)^{c} \cup\left(W^{*} \cap \mathbf{S}^{1}\right)^{c} \\
& =\left(\overline{\omega_{0}(W)} \cap \mathbf{S}^{1}\right) \cup\left(\bar{W} \cap \mathbf{S}^{1}\right)=\left(\overline{\omega_{0}(W)} \cup \bar{W}\right) \cap \mathbf{S}^{1}
\end{aligned}
$$

Hence $\overline{\omega_{0}(W)}$ and $\bar{W}$ cannot be disjoint. If $\overline{\omega_{0}(W)}=\bar{W}$, then $\overline{\omega_{0}(W)} \cup \bar{W}=\bar{W}$. From (4.3), we obtain $\mathbf{S}^{1}=\bar{W} \cup \mathbf{S}^{1}$. But $\bar{W}$ is a closed half-plane. This is absurd. We thus conclude that $\omega_{0}(W)$ and $W$ cannot be both maximal elements of $\mathcal{U}_{c}$. Lemma 4.3 is proved. 


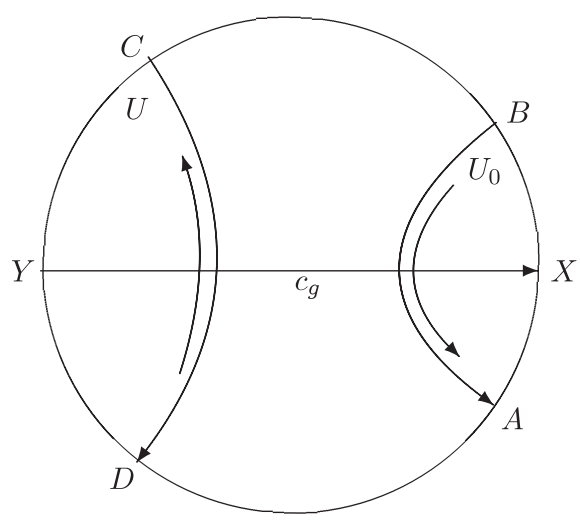

FIGURE 1. The axis $c_{g}$ and the sets $U$ and $U_{0}$.

\section{Boundaries of twice punctured disks on $S$ that enclose a fixed puncture}

In this section we handle the case in which the curve system $\mathcal{C}$ defined as in (3.1) contains a curve $c$ that is trivial on $\tilde{S}$. We begin with the following lemma.

Lemma 5.1. Suppose that $c_{g}$ crosses $\Sigma_{\alpha}$. Then as a circle homeomorphism, the map $\left.\tau_{\alpha}^{k} g\right|_{\mathbf{S}^{1}}$ does not fix any parabolic fixed point of $G$.

PROOF. By hypothesis, the axis $c_{g}$ meets $\Sigma_{\alpha}$. Let $U$ be a maximal element of $\mathcal{U}_{\alpha}$ such that $c_{g}$ intersects $\partial U$. Assume without loss of generality that $U$ covers the repelling fixed point $Y$ of $g$. There exists another maximal element $U_{0}$ of $\mathcal{U}_{\alpha}$ that contains $g(\mathbf{H}-U)$. Then, of course, the half-plane $U_{0}$ covers the attracting fixed point $X$ of $g$. Write $\{A, B\}=\partial U_{0} \cap \mathbf{S}^{1}$ and $\{C, D\}=\partial U \cap \mathbf{S}^{1}$, as labeled in Figure 1. Let $(A B)$ denote the circular arc connecting $A$ and $B$ on $\mathbf{S}^{1}$ without passing through any other labeled points.

Clearly, on $(Y C) \cup(C B) \cup(B X)$ the action of $g$ is consistent with the action of $\tau_{\alpha}$. Hence there are no fixed points of $\tau_{\alpha}^{k} g$ there. Let $z \in(D A)$. If $z$ is not covered by any maximal element of $\mathcal{U}_{\alpha}$, then $\tau_{\alpha}^{-k}(z)=z$ and thus $g^{-1} \tau_{\alpha}^{-k}(z)=g^{-1}(z) \neq z$. This implies that $\tau_{\alpha}^{k} g(z) \neq z$. If $z$ is covered by a maximal element $V$ of $\mathcal{U}_{\alpha}$, then $V$ is disjoint from $U$ and $U_{0}$, and $\tau_{\alpha}^{-k}(z) \in V \cap \mathbf{S}^{1}$. Observe that $\partial V$ also projects to $\tilde{\alpha}$, and $g^{-1}(V) \cap V=\emptyset$. It follows that $g^{-1} \tau_{\alpha}^{k}(z) \neq z$. We conclude that there are no fixed points of $\tau_{\alpha}^{k} g$ on $(D A)$.

We must show that $\tau_{\alpha}^{k} g$ has no fixed points on $(A X) \cup(D Y)$. Let $z \in(A X)$. By [15, Lemma 3.1], there exist a finite number of elements $U_{0}, U_{1}, \ldots, U_{m}$ in $\mathcal{U}_{\alpha}$ such that $U_{0} \supset U_{1} \supset \cdots \supset U_{m} \ni z$. This tells us that

$$
\epsilon\left(\tau_{\alpha}, z\right)=m+1 \text {. }
$$

Now $g(z)$ is covered by $U_{0} \supset g\left(U_{0}\right) \supset g\left(U_{1}\right) \supset \cdots \supset g\left(U_{m}\right) \ni g(z)$. By invariance, all $g\left(U_{i}\right) \in \mathcal{U}_{\alpha}$. Since $U_{0} \in \mathcal{U}_{\alpha}$, we see that $\epsilon\left(\tau_{\alpha}, g(z)\right) \geq m+2$. But $g(z) \in(A X)$, 
so $\epsilon\left(\tau_{\alpha}, \tau_{\alpha}^{k} g(z)\right)=\epsilon\left(\tau_{\alpha}, g(z)\right)$. Hence

$$
\epsilon\left(\tau_{\alpha}, \tau_{\alpha}^{k} g(z)\right)=\epsilon\left(\tau_{\alpha}, g(z)\right) \geq m+2 .
$$

Combining (5.1) and (5.2) leads to $\tau_{\alpha}^{k} g(z) \neq z$.

Similarly, by considering the inverse of the map $\tau_{\alpha}^{k} g$, one can prove that there are no fixed points of $\tau_{\alpha}^{k} g$ on $(D Y)$. The details are omitted.

Finally, we notice that any labeled point in $\{A, B, C, D, X, Y\}$ is a fixed point of a hyperbolic element of $G$; it cannot be a fixed point of any parabolic element of $G$. This proves Lemma 5.1.

Assume that for some integer $k$, the mapping class $\theta=\varphi^{*}\left(\left[\tau_{\alpha}^{k} g\right]\right)$ is reducible by a curve system (3.1). Let $f$ be a representative of $\theta$ such that

$$
f\left(\left\{c_{1}, \ldots, c_{s}\right\}\right)=\left\{c_{1}, \ldots, c_{s}\right\} .
$$

PROPOSITION 5.2. The system $\mathcal{C}$ does not contain any curve c that is trivial on $\tilde{S}$.

PROOF. If $\tilde{S}$ is compact, then there is nothing to prove. We assume henceforth that $\tilde{S}$ contains at least one puncture.

Suppose on the contrary that $\mathcal{C}$ contains a curve $c$ that is trivial on $\tilde{S}$. Then $c$ is the boundary of a twice punctured disk $\Delta$ enclosing $a$ and a puncture of $\tilde{S}$. We observe that any two punctured disks $\Delta_{1}$ and $\Delta_{2}$, if both enclose the puncture $a$, must have an overlap. This shows that $\partial \Delta_{1}$ intersects $\partial \Delta_{2}$. On the other hand, by definition, curves in $\mathcal{C}$ are mutually disjoint. We see that there is exactly one curve $c$ in $\mathcal{C}$ such that $\tilde{c}$ is trivial.

Now $f$ is a self-map of $S$ with $f(a)=a$, and the region $f(\Delta)$ must also be a twice punctured disk enclosing $a$. Then by the above argument, we obtain $\partial \Delta \cap \partial f(\Delta) \neq \emptyset$. Hence if $f(c) \neq c$, then $f(c) \notin \mathcal{C}$. So we must have $f(c)=c$.

Choose $[\omega] \in \bmod (\tilde{S})$ so that $\varphi^{*}([\omega])=\theta$ is represented by $f$. By Lemma 4.2, any representative $\omega_{0}$ of $[\omega]$ fixes a parabolic fixed point $x$ of $G$. On the other hand, we observe that $\varphi^{*}\left(\left[\tau_{\alpha}^{k} g\right]\right)=t_{\alpha}^{k} \circ \varphi^{*}(g)=\theta$. We see that $[\omega]=\left[\tau_{\alpha}^{k} g\right]=\left[\tau_{\alpha}^{k}\right] g$, and thus

$$
\left.\omega\right|_{\mathbf{S}^{1}}=\left.\tau_{\alpha}^{k} g\right|_{\mathbf{S}^{1}}
$$

From Lemma 5.1, we conclude that $\left.\tau_{\alpha}^{k} g\right|_{\mathbf{S}^{1}}$ does not fix any parabolic fixed point of $G$. It follows from (5.3) that $\left.\omega\right|_{\mathbf{S}^{1}}$ does not fix any parabolic fixed point of $G$, which leads to a contradiction.

\section{Proof of Theorem 1.1}

By hypothesis, $c_{g} \cap \Sigma_{\alpha} \neq \emptyset$. Let $U \in \mathcal{U}_{\alpha}$ be the maximal element such that $c_{g}$ intersects $\partial U$. Suppose that $\theta=\varphi^{*}\left(\left[\tau_{\alpha}^{k} g\right]\right)$ is reduced by (3.1). By Proposition 5.2, the curve system $\mathcal{C}$ does not contain any curve $c$ with $\tilde{c}$ trivial on $\tilde{S}$. Choose $c \in \mathcal{C}$. Then Lemma 3.2 leads to $\theta^{2}(c)=c$. By Lemma 3.3, we can assume that $c \in \Lambda_{1}$. Thus, by 


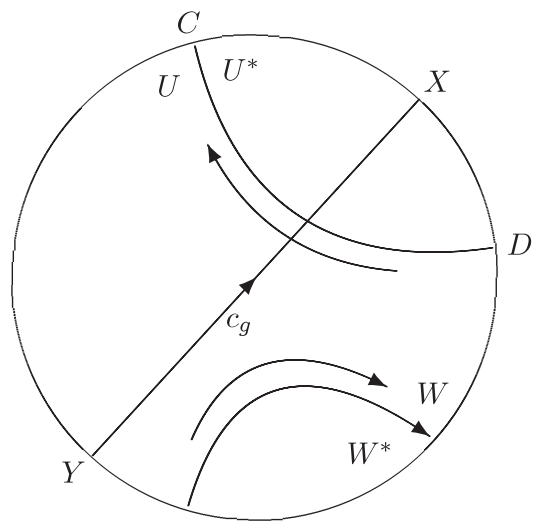

(a)

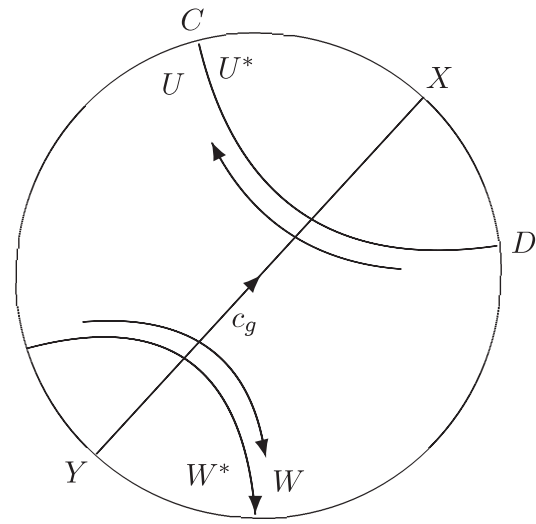

(b)

FIGURE 2. Two of the cases in the proof of Theorem 1.1.

Lemma 3.5, there exists a maximal element $W \in \mathcal{U}_{c}$ such that either $\partial U \cap \partial W=\emptyset$, $U \cap W \neq \emptyset$, and $U \cup W=\mathbf{H}$, or both $U$ and $U_{0}$ are contained in $W$, where $U_{0}$ is as in Lemma 3.5. If the former possibility occurs, we let $Y$ denote the intersection $c_{g} \cap\left(U \cap \mathbf{S}^{1}\right)$ and $X$ the intersection $c_{g} \cap\left((\mathbf{H}-U) \cap \mathbf{S}^{1}\right)$. There are four cases in total:

(i) $Y$ is the repelling fixed point of $g$ and $\partial W$ is disjoint from $c_{g}$;

(ii) $Y$ is the repelling fixed point of $g$ and $\partial W$ intersects $c_{g}$;

(iii) $Y$ is the attracting fixed point of $g$ and $\partial W$ is disjoint from $c_{g}$; and

(iv) $Y$ is the attracting fixed point of $g$ and $\partial W$ intersects $c_{g}$.

We only discuss the first two cases, which are drawn in Figures 2(a) and (b). The other two cases can be treated by considering the inverse of $\tau_{\alpha}^{k} g$.

Case (i). The geodesic boundary $\partial W$ is disjoint from $c_{g}$. In this case, the complement $W^{*}$ of $W$ is in $U$ (Figure 2(a)). Now $W^{*}$ is disjoint from $c_{g}$ to $W \in \mathcal{U}_{c}$ is maximal. Since $\partial W=\partial W^{*}$ projects to $\tilde{c}$ which is nontrivial on $\tilde{S}$, we see that $g\left(W^{*}\right) \cap W^{*}=\emptyset$. From Lemma 3.1, the geodesic $\tilde{c}$ is disjoint from $\tilde{\alpha}$. Since $\partial U$ projects to $\tilde{\alpha}$, either $g\left(W^{*}\right) \subset U$ or $g\left(W^{*}\right) \subset U^{*}$. If $g\left(W^{*}\right) \subset U^{*}$, then since $\tau_{\alpha}^{k}$ keeps $U^{*}$ invariant, we see that $\tau_{\alpha}^{k} g\left(W^{*}\right) \subset U^{*}$ and that $\left(\tau_{\alpha}^{k} g\right)^{2}\left(W^{*}\right) \subset U^{*}$. Hence $\left(\tau_{\alpha}^{k} g\right)^{2}\left(W^{*}\right) \cap W^{*}=\emptyset$. By Lemma 4.3, we conclude that $\left(\tau_{\alpha}^{k} g\right)^{2}(W)$ is not a maximal element of $\mathcal{U}_{c}$.

Assume that $g\left(W^{*}\right) \subset U$. Note that the Euclidean diameter of $W^{*}$ is positive and that $\partial W^{*}$ projects to $\tilde{c}$, and all boundaries of elements of $\mathcal{U}_{\alpha}$ project to $\tilde{\alpha}$. Since $\tilde{c}$ is disjoint from $\tilde{\alpha}$, all boundaries of elements of $\mathcal{U}_{\alpha}$ are disjoint from $\partial W^{*}$. We see that there are only finitely many elements

$$
U_{0}=U, U_{1}, \ldots, U_{r}
$$


of $\mathcal{U}_{\alpha}$ so that

$$
W^{*} \subset U_{r} \subset \cdots \subset U_{1} \subset U
$$

Let $\epsilon_{1}\left(\tau_{\alpha}, W^{*}\right)$ denote the number of elements of $\mathcal{U}_{\alpha}$ that cover $W^{*}$. By definition of $\tau_{\alpha}$, we know that for $i=0, \ldots, r$, the half-planes $\tau_{\alpha}^{-k}\left(U_{i}\right)$ are elements of $\mathcal{U}_{\alpha}$ and $\operatorname{cover} \tau_{\alpha}^{-k}\left(W^{*}\right)$. It follows that

$$
\epsilon_{1}\left(\tau_{\alpha}, \tau_{\alpha}^{-k}\left(W^{*}\right)\right) \geq \epsilon_{1}\left(\tau_{\alpha}, W^{*}\right) .
$$

Notice that $U$ and all $g^{-1} \tau_{\alpha}^{-k}\left(U_{i}\right)$, for $i=0, \ldots, r$, are elements of $\mathcal{U}_{\alpha}$. Since $g$ is a Möbius transformation, from (6.1) we obtain

$$
g^{-1} \tau_{\alpha}^{-k}\left(W^{*}\right) \subset g^{-1} \tau_{\alpha}^{-k}\left(U_{r}\right) \subset \cdots \subset g^{-1} \tau_{\alpha}^{-k}\left(U_{0}\right) \subset U
$$

From (6.2) along with (6.3), we assert that

$$
\epsilon_{1}\left(\tau_{\alpha}, g^{-1} \tau_{\alpha}^{-k}\left(W^{*}\right)\right)>\epsilon_{1}\left(\tau_{\alpha}, \tau_{\alpha}^{-k}\left(W^{*}\right)\right) \geq \epsilon_{1}\left(\tau_{\alpha}, W^{*}\right) .
$$

In particular, (6.4) yields that $\epsilon_{1}\left(\tau_{\alpha}, g^{-1} \tau_{\alpha}^{-k}\left(W^{*}\right) \neq \epsilon_{1}\left(\tau_{\alpha}, W^{*}\right)\right)$. Thus we must have $g^{-1} \tau_{\alpha}^{-k}\left(W^{*}\right) \neq W^{*}$. A similar argument yields that

$$
\epsilon_{1}\left(\tau_{\alpha},\left(g^{-1} \tau_{\alpha}^{-k}\right)^{2}\left(W^{*}\right)\right)>\epsilon_{1}\left(\tau_{\alpha}, W^{*}\right) .
$$

Thus $\left(g^{-1} \tau_{\alpha}^{-k}\right)^{2}\left(W^{*}\right) \neq W^{*}$.

If $\left(g^{-1} \tau_{\alpha}^{-k}\right)^{2}\left(W^{*}\right) \cap W^{*}=\emptyset$, by Lemma 4.3, the half-plane $\left(g^{-1} \tau_{\alpha}^{-k}\right)^{2}(W)$ is not a maximal element of $\mathcal{U}_{\alpha}$. If $\left(g^{-1} \tau_{\alpha}^{-k}\right)^{2}\left(W^{*}\right) \supset W^{*}$, then (6.5) is impossible. If $\left(g^{-1} \tau_{\alpha}^{-k}\right)^{2}\left(W^{*}\right) \subset W^{*}$, then $\left(g^{-1} \tau_{\alpha}^{-k}\right)^{2}(W) \supset W$. This says that if $\left(g^{-1} \tau_{\alpha}^{-k}\right)^{2}(W)$ were a maximal element of $\mathcal{U}_{c}$, then $W$ would not be a maximal element of $\mathcal{U}_{c}$. It follows that $\left(g^{-1} \tau_{\alpha}^{-k}\right)^{2}(W)$ is not maximal. But this contradicts Lemma 4.1.

Case (ii). The geodesic boundary $\partial W$ intersects $c_{g}$. In this case, $W^{*} \subset U$; see Figure 2(b). Since $Y$ is the attracting fixed point of $g$ that is covered by $W^{*}$, we have $W^{*} \subset g\left(W^{*}\right)$.

If $U \subseteq g\left(W^{*}\right)$, then $g(W) \subseteq U^{*} \subset W$. Since $\tau_{\alpha}^{k}$ keeps $U^{*}$ invariant, we have $\tau_{\alpha}^{k} g(W) \subseteq U^{*} \subset W$. Since $X$ is the attracting fixed point of $g$ that is covered by $U^{*}$, we must have $g \tau_{\alpha}^{k} g(W) \subseteq U^{*} \subset W$. Hence, $\tau_{\alpha}^{k} g \tau_{\alpha}^{k} g(W)=\left(\tau_{\alpha}^{k} g\right)^{2}(W) \subseteq U^{*} \subset W$, which says that $\left(\tau_{\alpha}^{k} g\right)^{2}(W)$ is not a maximal element of $\mathcal{U}_{c}$.

If $g\left(W^{*}\right) \subset U$, then since $U$ is a maximal element of $\mathcal{U}_{\alpha}$, the map $\tau_{\alpha}^{k}$ keeps $U$ invariant. Hence $\tau_{\alpha}^{k} g\left(W^{*}\right) \subset U$. Clearly, $\tau_{\alpha}^{k} g\left(W^{*}\right) \cap g\left(W^{*}\right)=\emptyset$. Now we can also easily check that $\left(\tau_{\alpha}^{k} g\right)^{2}\left(W^{*}\right) \cap g\left(W^{*}\right)=\emptyset$. By Lemma 4.3, we see that $\left(\tau_{\alpha}^{k} g\right)^{2}(W)$ is not a maximal element of $\mathcal{U}_{c}$.

Finally, if $W$ contains both $U$ and $U_{0}$, then $c_{g} \subset W$ and $W^{*}$ is disjoint from $c_{g}$ and $U$. In this case, we use the same argument as in Case (i) above to conclude that $\left(\tau_{\alpha}^{k} g\right)^{2}(W)$ is not a maximal element of $\mathcal{U}_{c}$. 


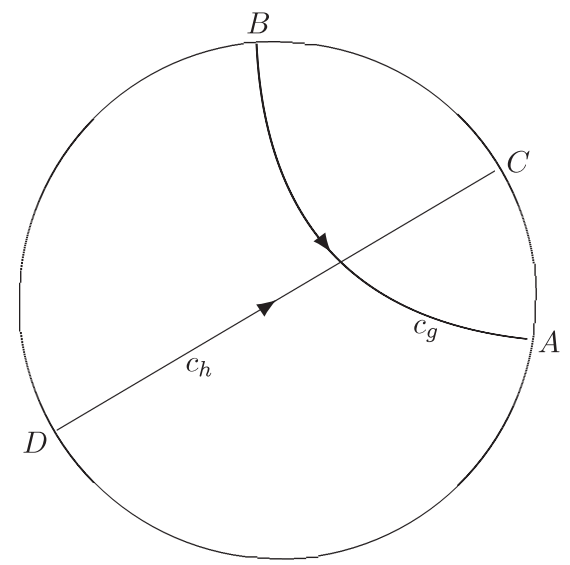

FIGURE 3. Hyperbolic elements whose axes intersect.

\section{Proof of Corollary 1.2}

We first prove the following result.

Lemma 7.1. Let $g, h \in G$ be hyperbolic elements. Assume that their axes $c_{g}$ and $c_{h}$ intersect. Then for all integers $r$ and $s$, the elements $h^{r} g^{s} \in G$ are also hyperbolic, and their axes intersect both $c_{h}$ and $c_{g}$.

Proof. Let $\{A, B\}$ and $\{C, D\}$ denote the fixed points of $g$ and $h$, respectively, where $A$ and $C$ are the attracting fixed points and $B$ and $D$ are the repelling fixed points; see Figure 3.

We assume that both $r$ and $s$ are positive. Consider the motion of $\xi=h^{r} g^{s}$ on $\mathbf{S}^{1}$. Notice that $A$ and $C$ are the attracting fixed points of $g^{s}$ and $h^{r}$ respectively, and $B$ and $D$ are the repelling fixed points of $g^{s}$ and $h^{r}$ respectively. We also observe that the motion $\xi$ at $A$ is toward $C$ in the counterclockwise direction, and at $C$ is toward $A$ in the clockwise direction. Similarly, the motion $\xi$ at $B$ is toward $C$ in the clockwise direction, and at $D$ is toward $A$ in the counterclockwise direction. Therefore, by calculus there is an attracting fixed point $X$ for the motion $\xi$ in the $\operatorname{arc}(A C)$ and a repelling fixed point $Y$ for the motion $\xi$ in the $\operatorname{arc}(B D)$ (not shown in Figure 3). Since $\xi$ is a Möbius transformation, it has at most two fixed points on $\mathbf{S}^{1}$. It follows that $\xi$ has exactly two fixed points $X$ and $Y$. We conclude that $\xi$ is hyperbolic and its axis $c_{\xi}$ is the geodesic connecting $X$ and $Y$.

Since $X$ and $Y$ lie on different sides of $c_{h}$, we see that $c_{\xi}$ intersects $c_{h}$. Similarly, we note that $X$ and $Y$ also lie on different sides of $c_{g}$. Thus $c_{\xi}$ also intersects $c_{g}$. Therefore $c_{\xi}$ intersects both $c_{h}$ and $c_{g}$.

From Lemma 7.1, we conclude that the axis $c_{h g}$ of $h g$ intersects $c_{h}$. In particular, this implies that $c_{h g} \cap \Sigma_{\alpha} \neq \emptyset$ and $c_{h g} \cap \Sigma_{\alpha_{0}} \neq \emptyset$. Hence by Theorem 1.1 we see that 
$t_{\alpha} \circ \varphi^{*}(h g)$ is pseudo-Anosov. But since $\varphi^{*}(h)=t_{\alpha}^{-1} \circ t_{\alpha_{0}}$, we obtain

$$
t_{\alpha} \circ \varphi^{*}(h g)=t_{\alpha} \circ\left(t_{\alpha}^{-1} \circ t_{\alpha_{0}}\right) \circ \varphi^{*}(g)=t_{\alpha_{0}} \circ \varphi^{*}(g) .
$$

Hence $t_{\alpha_{0}} \circ \varphi^{*}(g)$ is pseudo-Anosov.

To prove that $t_{\alpha}^{-1} \circ \varphi^{*}(g)$ pseudo-Anosov, we use Theorem 1.1 once again. By assumption, $c_{h g} \cap \Sigma_{0} \neq \emptyset$. So Theorem 1.1 asserts that $t_{\alpha_{0}}^{-1} \circ \varphi^{*}(h g)$ is pseudoAnosov. A computation shows that

$$
t_{\alpha_{0}}^{-1} \circ \varphi^{*}(h g)=t_{\alpha}^{-1} \circ \varphi^{*}(g) .
$$

Hence $t_{\alpha}^{-1} \circ \varphi^{*}(g)$ is pseudo-Anosov. This proves Corollary 1.2.

\section{Examples}

In this section we give an example to show that Theorem 1.1 is no longer true if we drop the assumption that $c_{g} \cap \Sigma_{\alpha} \neq \emptyset$. We take a simple closed geodesic $\alpha$ on $S$ that is also nontrivial on $\tilde{S}$. Let $f \in \mathcal{F}(S, a)$ be an arbitrary element. Then it is well known (see Masur and Minsky [10], for example) that for a sufficiently large integer $k$, the pair $\left\{\alpha, f^{k}(\alpha)\right\}$ fills $S$. Denote by $\beta$ the geodesic homotopic to $f^{k}(\alpha)$. From Thurston [14], for any positive integer $i$, the mapping class $\theta_{i}$ induced by

$$
t_{\alpha}^{-i} \circ t_{\beta}^{i}
$$

is pseudo-Anosov. Since it also projects to the identity on $\tilde{S}$, by [7, Theorem 2], there is an essential hyperbolic element $g_{i} \in G$ such that $\varphi^{*}\left(g_{i}\right)=\theta_{i}$ that is represented by $(8.1)$.

Let $\left[\tau_{\alpha}\right],\left[\tau_{\beta}\right] \in \bmod (\tilde{S})$ be such that $\varphi^{*}\left(\left[\tau_{\alpha}\right]\right)=t_{\alpha}$ and $\varphi^{*}\left(\left[\tau_{\beta}\right]\right)=t_{\beta}$. By the same argument as Lemma 3.4, there exist a maximal element $U$ of $\mathcal{U}_{\alpha}$ and a maximal element $V$ of $\mathcal{U}_{\beta}$ such that $U \cap V \neq \emptyset, \partial U \cap \partial V=\emptyset$, and $U \cup V=\mathbf{H}$. In particular, it follows that the region

$$
\Sigma_{0}=\mathbf{H}-\left\{\text { all maximal elements of } \tau_{\alpha} \text { and } \tau_{\beta}\right\}
$$

is empty. Now from [17, Theorem 1.2], the axis $c_{i}$ of $g_{i}$ stays in the region $U \cap V$. Since $U \cup V=\mathbf{H}$, the axis $c_{i}$ does not cross $\Sigma_{\alpha}$ and $\Sigma_{\beta}$ (defined as in (1.2)). This implies that $c_{i} \cap \Sigma_{\alpha}=\emptyset$ and $c_{i} \cap \Sigma_{\beta}=\emptyset$ (certainly, the geodesic $c_{i}$ intersects some nonmaximal elements of $\tau_{\alpha}$ and $\tau_{\beta}$ since $\tilde{c}_{i}$ is a filling closed geodesic that intersects $\tilde{\alpha}=\tilde{\beta})$. Now if we choose $k=i$ and consider the mapping class $t_{\alpha}^{k} \circ \varphi^{*}\left(g_{i}\right)$, then from (8.1), we obtain

$$
t_{\alpha}^{i} \circ \varphi^{*}\left(g_{i}\right)=t_{\alpha}^{i} \circ\left(t_{\alpha}^{-i} \circ t_{\beta}^{i}\right)=t_{\beta}^{i} .
$$

So for any integer $i$, the mapping class $t_{\alpha}^{i} \circ \varphi^{*}\left(g_{i}\right)$ is not pseudo-Anosov.

\section{Acknowledgements}

I am grateful to the referees for valuable comments, suggestions and encouragement. 


\section{References}

[1] M. Bauer, 'Examples of pseudo-Anosov homeomorphisms', Trans. Amer. Math. Soc. 330 (1992), 333-370.

[2] L. Bers, 'Fiber spaces over Teichmüller spaces', Acta Math. 130 (1973), 89-126.

[3] S. Boyer, C. Gordon and X. Zhang, 'Dehn fillings of large hyperbolic 3-manifolds', J. Differential Geom. $\mathbf{5 8}$ (2001), 263-308.

[4] A. Fathi, 'Dehn twists and pseudo-Anosov diffeomorphisms', Invent. Math. 87 (1987), 129-151.

[5] A. Fathi, F. Laudenbach and V. Poenaru, 'Travaux de Thurston sur les surfaces', in: Seminaire Orsay, Asterisque, Soc. Math. de France, 66-67 (1979), 1-284.

[6] N. V. Ivanov, 'Mapping class groups', Manuscript, 1998.

[7] I. Kra, 'On the Nielsen-Thurston-Bers type of some self-maps of Riemann surfaces', Acta Math. 146 (1981), 231-270.

[8] D. D. Long, 'Constructing pseudo-Anosov maps', in: Knot Theory and Manifolds, Lecture Notes in Mathematics, 1144 (Springer, Berlin, 1985), pp. 108-114.

[9] D. D. Long and H. Morton, 'Hyperbolic 3-manifolds and surface homeomorphism', Topology 25 (1986), 575-583.

[10] H. Masur and Y. Minsky, 'Geometry of the complex of curves I: Hyperbolicity', Invent. Math. 138 (1999), 103-149.

[11] S. Nag, 'Non-geodesic discs embedded in Teichmüller spaces', Amer. J. Math. 104 (1982), 339-408.

[12] R. C. Penner, 'The action of the mapping class group on isotopy classes of curves and arcs in surfaces', Thesis, MIT, 1982.

[13] R. C. Penner, 'A construction of pseudo-Anosov homeomorphisms', Proc. Amer. Math. Soc. 104 (1988), 1-19.

[14] W. P. Thurston, 'On the geometry and dynamics of diffeomorphisms of surfaces', Bull. Amer. Math. Soc. (N.S.) 19 (1988), 417-431.

[15] C. Zhang, 'Pseudo-Anosov maps and fixed points of boundary homeomorphisms compatible with a Fuchsian group', Osaka J. Math. 46 (2009), 783-798.

[16] C. Zhang, 'Singularities of quadratic differentials and extremal Teichmüller mappings defined by Dehn twists', J. Aust. Math. Soc. 3 (2009), 275-288.

[17] C. Zhang, 'Pseudo-Anosov mapping classes and their representations by products of two Dehn twists', Chinese Ann. Math. Ser. B 30 (2009), 281-292.

\section{ZHANG, Department of Mathematics, Morehouse College, Atlanta, GA 30314, USA \\ e-mail: czhang@morehouse.edu}

